\title{
Notch controls urothelial integrity in the mouse bladder
}

\author{
Varvara Paraskevopoulou, ${ }^{1,2}$ Vangelis Bonis, ${ }^{1}$ Vasilis S. Dionellis, ${ }^{3,4}$ Nikolaos Paschalidis, ${ }^{1}$ \\ Pelagia Melissa, ${ }^{1}$ Evangelia Chavdoula, ${ }^{1}$ Eleni Vasilaki, ${ }^{1}$ Ioannis S. Pateras, ${ }^{4}$ and Apostolos Klinakis ${ }^{1}$ \\ 'Biomedical Research Foundation of the Academy of Athens, Athens, Greece. 'Laboratory of Biology, School of Medicine, \\ National and Kapodistrian University of Athens, Athens, Greece. ${ }^{3}$ Department of Molecular Biology, University of Geneva, \\ Geneva, Switzerland. ${ }^{4}$ Laboratory of Histology-Embryology, Molecular Carcinogenesis Group, National and Kapodistrian \\ University of Athens, Athens, Greece.
}

The Notch signaling pathway mediates cell-cell communication regulating cell differentiation and proliferation and cell fate decisions in various tissues. In the urinary bladder, Notch acts as a tumor suppressor in mice, while mutations in Notch pathway components have been identified in human bladder cancer as well. Here we report that the genetic inactivation of Notch in mice leads to downregulation of cell-cell and cell-ECM interaction components, including proteins previously implicated in interstitial cystitis/bladder pain syndrome (IC/BPS), structural defects and mucosal sloughing, inflammation, and leaky urine-blood barrier. Molecular profiling of ailing mouse bladders showed similarities with IC/BPS patient tissue, which also presented low Notch pathway activity as indicated by reduced expression of canonical Notch targets. Urothelial integrity was reconstituted upon exogenous reactivation of the Notch pathway, implying a direct involvement of Notch. Despite damage and inflammation, urothelial cells failed to proliferate, uncovering a possible role for $\alpha_{4}$ integrin in urothelial homeostasis. Our data uncover a broad role for Notch in bladder homeostasis involving urothelial cell crosstalk with the microenvironment.

Conflict of interest: The authors have declared that no conflict of interest exists.

Copyright: ( 2020 , American Society for Clinical Investigation.

Submitted: September 4, 2019 Accepted: December 30, 2019 Published: February 13, 2020.

Reference information: /CI Insight. 2020;5(3):e133232.

https://doi.org/10.1172/jici.

insight.133232.

\section{Introduction}

The Notch signaling pathway is a highly conserved network of receptors, ligands, and effector proteins that mediate the crosstalk of neighboring cells and control important cell processes such as proliferation, differentiation, and apoptosis (1). It is thus implicated in normal development and tissue homeostasis and upon deregulation in disease (2). Contrasting roles of Notch signaling have been described in tumorigenesis, where Notch $\AA$ can act in a context-dependent manner either as an oncogene or a tumor suppressor $(3,4)$. Recent studies have identified frequent mutations within Notch pathway components in epithelial cancers $(5,6)$, while mouse studies have revealed a tumor suppressor role for the Notch pathway in urothelial carcinoma $(7,8)$. The urothelium is a specialized epithelium that lines the luminal surface of the urinary tract, which includes the renal pelvis, the ureters, the urethra, and the glandular ducts of the prostate. The role of the urothelial lining is to generate the tightest and most impermeable barrier in the body, the urine-blood barrier, which prevents ions, solutes, water and pathogens from crossing into the bloodstream (9). In the bladder, the barrier must endure serious size changes in urothelial surface during cycles of filling and voiding. Fully differentiated superficial cells, also called umbrella cells, in the apical surface of the urothelial lining are responsible for the barrier function. Superficial cells are polarized and their apical surface is composed of asymmetric unit membrane plaques and intervening hinges that form a continuous, highly impermeable surface (9). The basolateral aspect of superficial cells is populated by tight junctions (TJs), adherens junctions, and desmosomes $(9,10)$. TJs participate in paracellular movement and maintain superficial cell polarity (11). Molecular analysis of human and animal bladder tissue samples has indicated that reduced expression of the TJ protein zonula occludens-1 (ZO-1) (12) as well as several extracellular matrix (ECM) proteoglycans, such as chondroitin sulfate, perlecan, biglycan, and decorin, is associated with human and feline interstitial cystitis (IC) $(13,14)$, highlighting the importance of cell-cell and cell-ECM interactions in urothelial integrity and barrier functionality. Mouse studies have uncovered roles for ZO-1 in normal intestinal cell shedding and intestinal barrier reestablishment (15), while loss of $\alpha_{\mathrm{v}} \beta_{6}$ integrin leads to enterocyte shedding and compromised intestinal barrier (16). IC/bladder pain syndrome (IC/BPS) is a chronic inflammatory 
condition of the urinary bladder associated with suprapubic pain related to bladder filling accompanied by other symptoms, such as frequency and nocturia, in the absence of urinary tract infection and other obvious pathology (17). However, the underlying molecular mechanism of IC/BPS is still unclear while treatment is largely palliative. It is widely accepted that defects in urothelial integrity compromise the barrier and lead to chronic inflammation and pain.

Here we present for the first time to our knowledge evidence linking the activity of the Notch signaling pathway with the transcriptional control of various cell-cell and cell-ECM components. Genetic inactivation of the pathway in vivo in mouse bladders leads to defects in the umbrella layer integrity, mucosal sloughing, and inflammation. Expression analysis of Notch-deficient mouse bladder tissue revealed molecular similarities, including downregulation of Notch canonical targets, with human IC/BPS expression profiles.

\section{Results}

Notch inactivation leads to proliferative and structural abnormalities in the mouse bladder. We have previously shown that the normal mouse urothelium ubiquitously expresses Notch-1 and Notch- 2 receptors, the activated forms of which are found in the nucleus, most likely in response to JAG1 activation (7). To genetically abrogate Notch signaling in the mouse urothelium in an inducible manner, we used a CreERT2 transgene under the control of keratin 5 (Krt5) regulatory sequences $\left(K r t 5^{\text {CreERT2 }}\right)$, which drives Cre-dependent recombination in Krt5-expressing cells (18). We and others have shown that in the bladder, recombination is restricted to the basal layer of the urothelium $(19,20)$. $K r t 5^{\text {CreERT2 }}$ mice were crossed with mice carrying conditional knockout alleles of either the nicastrin locus $\left(N c s t n^{t}\right)(21)$ encoding a $\gamma$-secretase component or the RBPJ locus $\left(R B P J^{t}\right)$ (22) encoding the recombining binding protein suppressor of hairless, which provides to the Notch complex its DNA binding activity. All breeding programs also included an inducible Tomato-expressing transgene $\left(R 26^{\text {tom }}\right)(23)$, which allows visualization of cells in which recombination occurred (throughout the article all analyzed mice include at least 1 copy of the $R 26^{\text {tom }}$, which will not be mentioned in the respective genotypes).

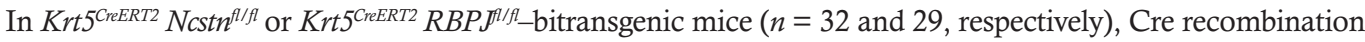
was induced through intraperitoneal injection (IP) of tamoxifen at the age of 6 weeks. Notch inactivation led to urothelial hyperplasia as early as 2 weeks following tamoxifen injection with occasional development of focal dysplasia (Figure 1A). Notch inactivation was supported with immunohistochemistry with antibodies against the intracellular domain of NOTCH 1 (Supplemental Figure 1A; supplemental material available online with this article; https://doi.org/10.1172/jci.insight.133232DS1). In agreement with our previous findings (7), Notch loss led to dual specificity phosphatase 4 downregulation and elevated phosphorylated ERK1/2 levels (Supplemental Figure 1, A and B). Mice also presented a previously unappreciated phenotype, which was highlighted by loss of KRT20 polarity and mucosal sloughing (Figure 1B), the extent of which correlated with the degree of recombination, as indicated by Tomato fluorescence. A similar phenotype, albeit less pronounced, was observed in bitransgenic mice ( $n=15$ and 20, respectively, for $K r t 14^{\text {creERT2 }} N_{c s t n}^{f l / f l}$ and Krt14 ${ }^{\text {CreERT2 }} R B P J^{\text {Ilfl }}$ mice) carrying our previously described (20) Krt14 locus-driven (Krt14 ${ }^{\text {CreERT2 }}$ CreERT2 transgene, which drives recombination in a progenitor population within the Krt5-expressing basal layer of the mouse urothelium (Supplemental Figure 2A). We were unable to monitor Notch-deficient mice carrying either Cre recombinase driver for longer than 1 month after tamoxifen injection because of severe skin abnormalities (Supplemental Figure 2B). In agreement with the in vivo hyperplasia, ex vivo assays indicated that Notch loss led to increased explant growth (Figure 1C).

To investigate whether the observed mucosal sloughing was exclusively linked to Notch loss from basal urothelial cells, we crossed $N c s t n^{f}$ and $R B P J^{f}$ mice with yet another Cre driver under the control of the $K r t 8$ locus regulatory sequences ( $K r t 8^{C r e R T 2}$ ) (24) expressing CreERT2 primarily in the superficial layer with occasional leakiness in intermediate and basal cells. Similar to that observed with the basal cell Cre drivers, Notch abrogation in $K r t 8^{\text {CreERT2 }} N c s t n^{f l f l}$ or $K r t 8^{\text {CreERT2 }} R B P J^{l / f l}$ ( $n=21$ and 14, respectively) mice led to hyperplasia, loss of KRT20 apical staining, and mucosal sloughing (Figure 1D). In humans, superficial cell sloughing can be the result of in situ carcinoma characterized by KRT20 positivity throughout the lesion (25). In this case, however, KRT20 positivity was restricted to a single superficial layer (Figure 1, B and D).

Increased inflammation of Notch-deficient mouse bladders. In the majority of cases, mucosal sloughing was accompanied by profound inflammation as indicated by CD45 immunofluorescence (Figure 2A) and mass cytometry (CyTOF) analysis (Figure 2B), while no apparent bacterial infection was detected. CD11b is a common myeloid marker (integrin $\alpha \mathrm{M}$ chain, also expressed by other leukocytes, especially of the myeloid lineage, such as dendritic cells). CD11b expression is highly activation dependent and represents 
A

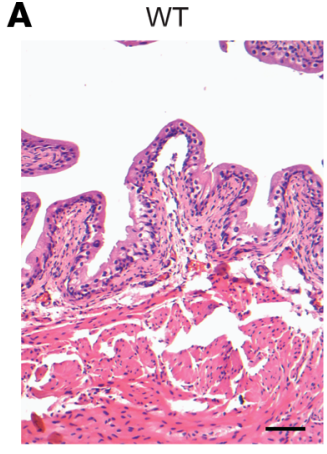

B $K r t 5^{\text {CreERT2 }} ; R 26^{\text {Tomato }}$

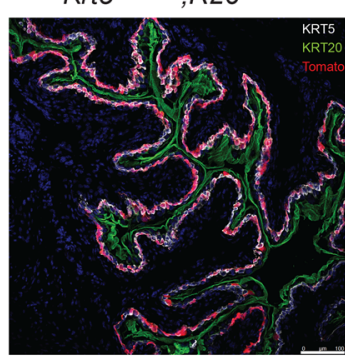

C
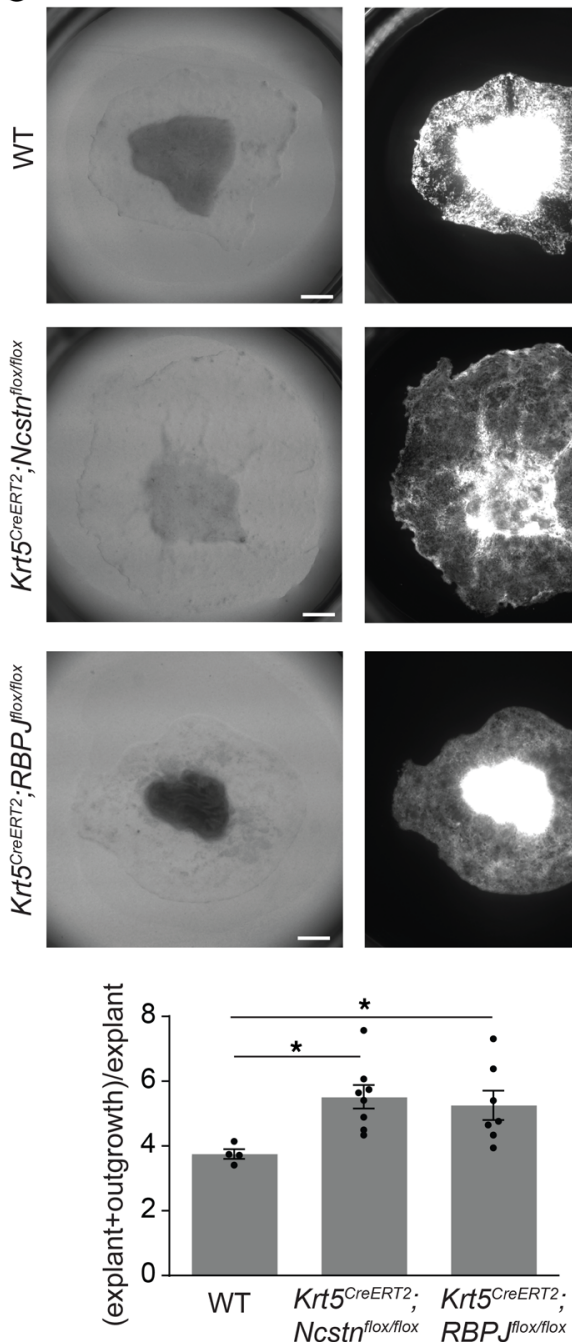

Krt5 ${ }^{\text {CreERT2 }} ;$ NCStn $^{\text {floxfllox }}$

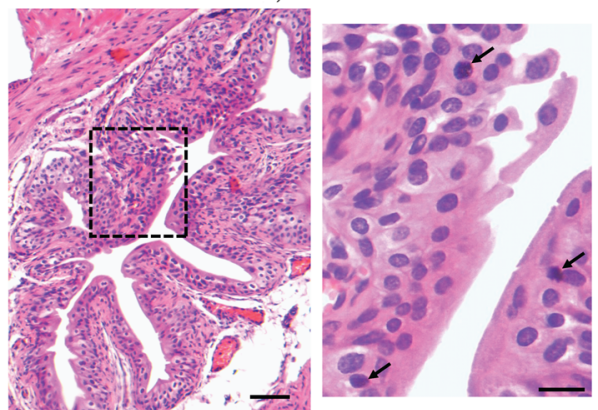

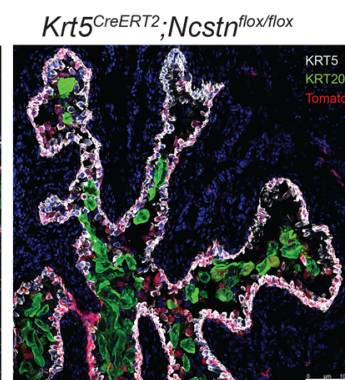
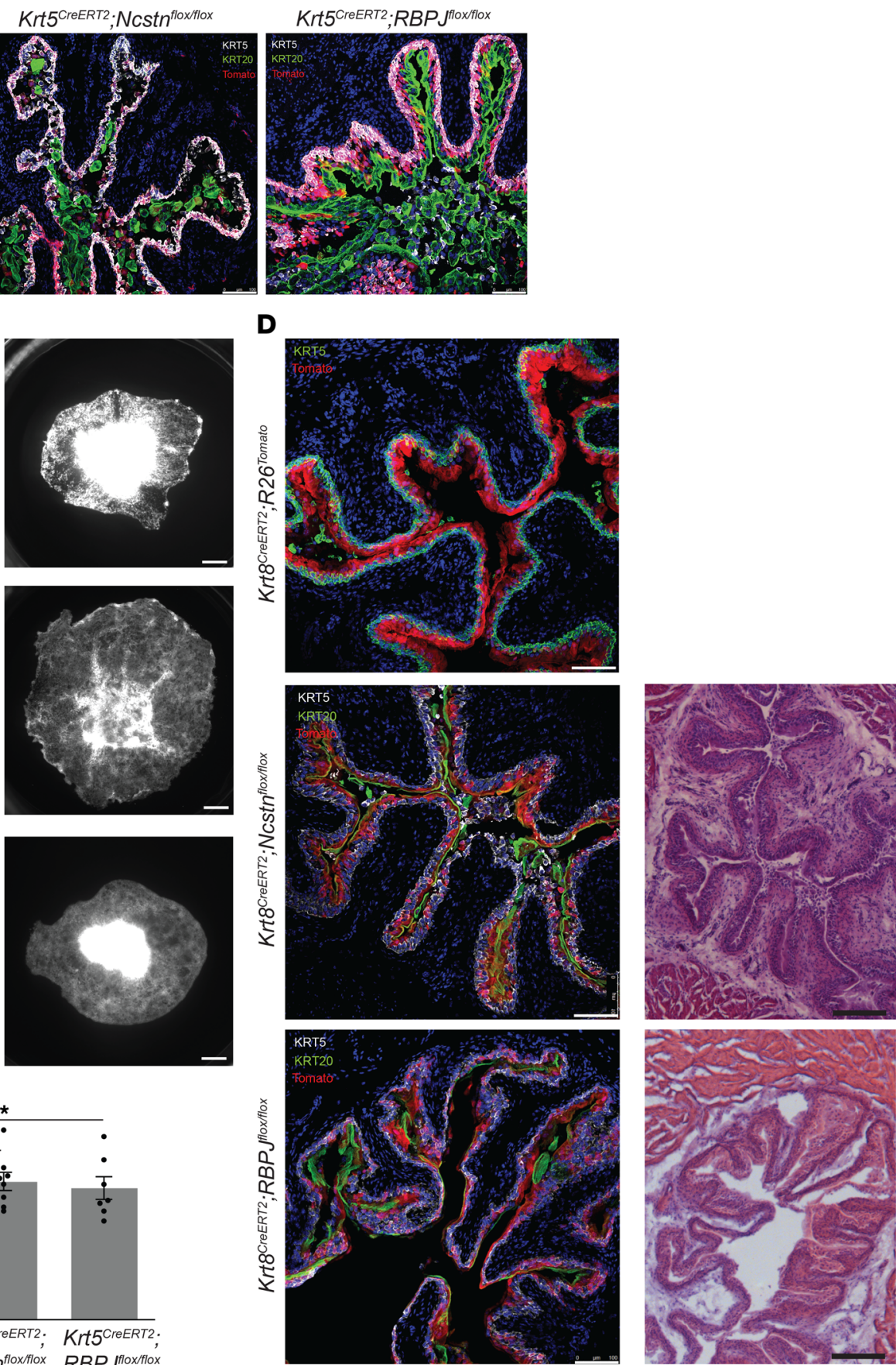
Figure 1. Notch inactivation leads to bladder hyperplasia and mucosal sloughing. (A) H\&E staining of bladder sections from WT (Krt5 $5^{\text {CreERT2 }} R 26^{\text {Tomato }}$ ) and

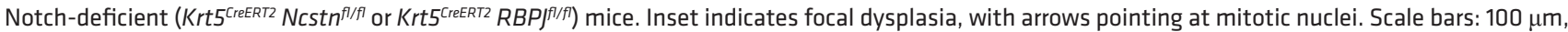
$50 \mu \mathrm{m}$ (inset). (B) Immunofluorescence on bladder sections from the same genotypes with the indicated antibodies. Scale bars: $100 \mu \mathrm{m}$. (C) Whole-mount bright-field and fluorescence images (Tomato) as well as scatter dot plot indicating growth from tissue explants from the same genotypes grown ex vivo for 7 days. Scale bars: $1 \mathrm{~mm}$. For growth calculation, the total area (explant plus outgrowth) was calculated with ImageJ software (NIH) and divided by the

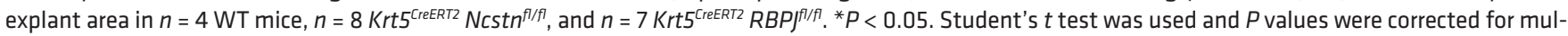
tiple testing using the Benjamini-Hochberg method. (D) H\&E and immunofluorescence on bladder sections from WT (Krt8 ${ }^{\text {CreERT2 }}$ R26 $^{\text {Tomato }}$ ), Krt8 ${ }^{\text {CreeRT2 }}$ Ncstn $^{\text {fl/fl }}$, or Krt8 $8^{\text {CreERT2 }} R B P f^{f / f l}$ mice with the indicated antibodies. Scale bars: $100 \mu \mathrm{m}$. DAPI was used as a nuclear counterstain in B and $\mathbf{D}$. Student's $t$ test was used.

a well-described marker of acute and chronic inflammation in mice and humans (26). The infiltration of CD $11 b^{\text {hi }}$ cells (Figure 2B) in $K r t 5^{\text {CreERT2 }} N c s t n^{f / f l}$ cells implies an active inflammatory response in response to Notch abrogation. Of interest, experimental mice showed mast cell infiltration (Figure 2C), a phenotype that in humans is associated with IC $(27,28)$.

Molecular profiling of Notch-deficient mouse bladders reveals molecular similarities with IC/BPS patients. To gain insight into the molecular basis of the loss of urothelial integrity and mucosal sloughing, we performed RNA-Seq experiments using $K r t 5^{\text {CreERT2 }} N_{c s t n^{f / f l}}$ mice. Gene Ontology (GO) analysis indicated that the top 20 pathways affected were overwhelmingly populated by inflammation- and epithelial integrity-related ones (Figure 3A). Enriched GO networks based on the differentially (primarily downregulated) expressed transcripts indicated 3 major hubs: ECM organization/extracellular structure organization, epithelial cell proliferation, and regulation of vasculature development/regulation of angiogenesis (Supplemental Figure 3). Of interest, RNA-Seq experiments on chemical-induced rat models of IC indicate that processes and pathways associated with ECM organization, blood vessel formation, antigen processing and presentation, immune response, and others are similarly affected (29).

Vasculature/angiogenesis defects were supported by microscopic analysis, indicating that Notch-deficient mouse bladders presented smaller blood vessels in comparison with WT controls (Supplemental Figure 4). Although impaired vasculature/angiogenesis could have a causal role in the loss of urothelial integrity, this is rather unlikely because the 2 phenotypes appeared simultaneously as early as 2 weeks after tamoxifen administration. Comparison of the networks affected in $K r t 5^{\text {CreERT2 }}$ $N c s t n^{f l f l}$ mice and human biopsies from patients with IC indicated that the majority (34/62) of deregulated processes in $K r t 5^{\text {CreERT2 }} \mathrm{Ncstn}^{f l f l}$ mice were also deregulated in nonulcerative and ulcerative bladder regions of patients with IC (30) (Figure 3B). More importantly, the expression of canonical Notch signaling targets HEY1, HES1, HES4, and DTX4 was reduced in both nonulcerative and ulcerative tissue in comparison with healthy tissue (Figure 3C). Although it is unclear whether the cause of the mouse and human condition is common, our data indicate that the molecular profiles of affected tissues share similarities, including the downregulation of Notch target genes.

In support of this, proteoglycan members previously associated with IC $(13,14)$ such as perlecan (Hspg2), components of TJs (Jam2, Jam3, and Cldn11), integrins (Itga4, Itga9, Itgb8, and others), and ECM proteins (fibronectin, tenascin, collagens, and thrombospondin) were downregulated in the RNA-Seq data (Figure 3D). Quantitative PCR analysis supported the RNA-Seq data showing downregulation of several integrins and IC markers, such as proteoglycans and ZO-1, upon loss of Notch signaling by means of Ncstn or RBPJ knockout in either the basal or the umbrella layer (Figure 3, E and F). Overall, our molecular and phenotypic analysis uncovers a possible link between the loss of Notch activity and the development of IC/BPS.

The concomitant downregulation of several cell-cell and cell-ECM components could lead to compromised urothelial integrity and explain the observed mucosal sloughing. To investigate whether these changes likely affect bladder function and lead to a defective urine-blood barrier, we measured urea levels in the blood serum of experimental and WT mice. Overall, loss of Notch activity was associated with uremia, a phenotype most obvious in Krt8CreERT2 Ncstn ${ }^{f l / f}$ mice (Figure 3G). This is in agreement with previous reports implicating TJ proteins in intestinal permeability (31). Interestingly, $\alpha_{\mathrm{v}} \beta_{6}$ integrin, which is required for maintaining intestinal barrier function (16), is downregulated in patients with inflammatory bowel disease (IBD) (32). In addition, loss of the hemidesmosome $\alpha_{6} \beta_{4}$ integrin leads to loss of epithelial integrity and denudation (33-35), while inactivation of $\beta_{1}$ integrin, the partner of $\alpha_{4}$ integrin, in mice leads to neonatal lethality from dehydration due to defects in the skin barrier (36). These data underscore the importance of cell-cell and cell-ECM interactions in tissue integrity and function in epithelia. In the case of epithelial barriers, defects in barrier function followed by increased permeability lead to a condition strongly associated with inflammation. In this aspect, it is interesting that patients with IBD also present with increased intestinal barrier permeability. 
A

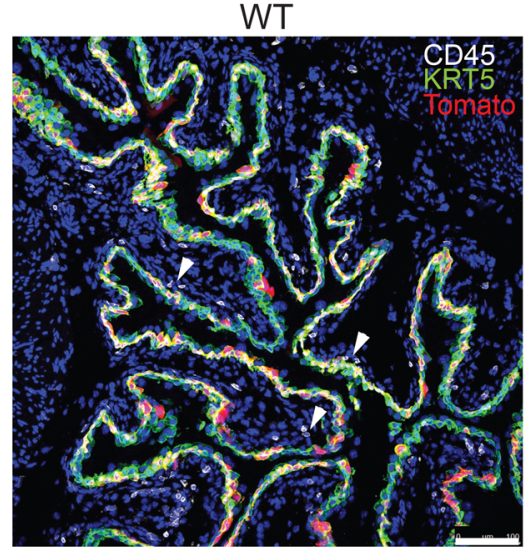

Krt5 ${ }^{\text {CreERT2 }} ;$ NCsth $^{\text {floxflox }}$

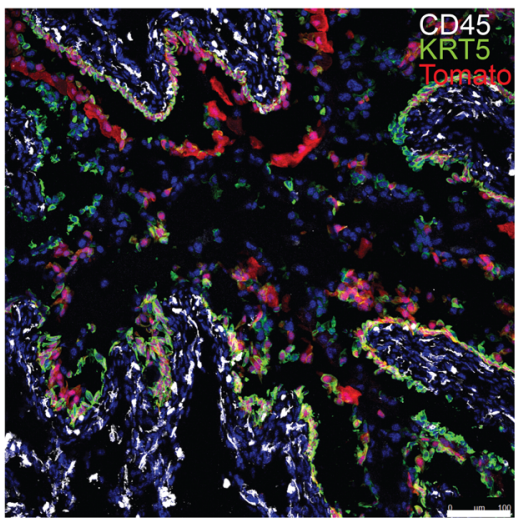

$K r+5^{\mathrm{CreERT2}}: R B \mathrm{~J}_{\text {flox/flox }}$

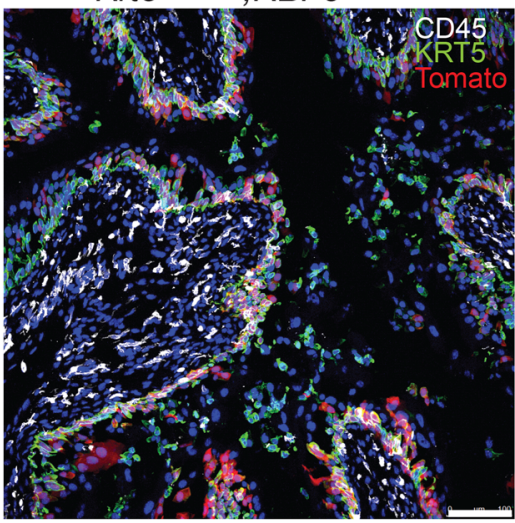

B

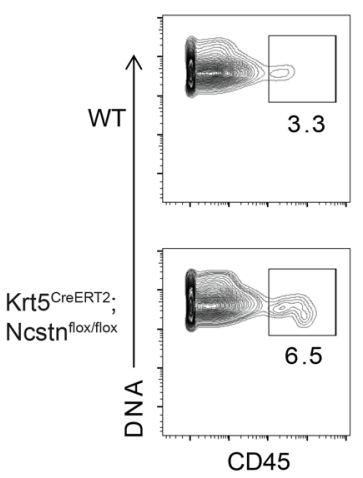

C
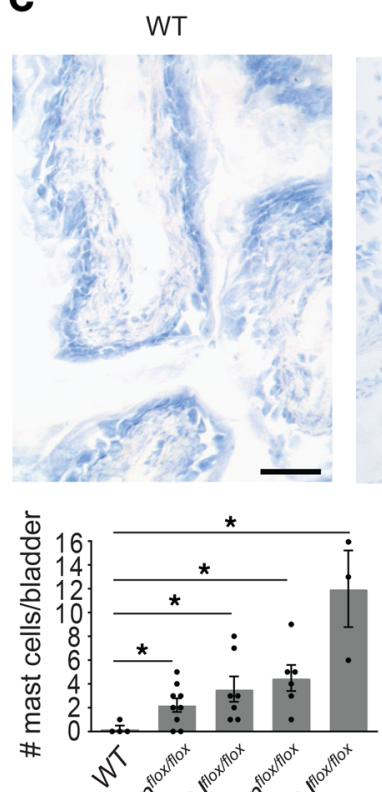

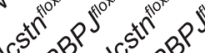

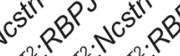

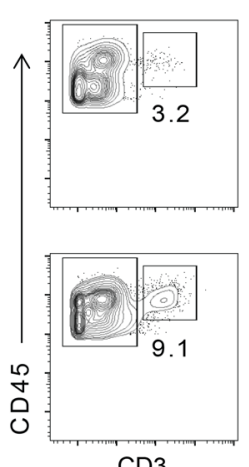

CD3
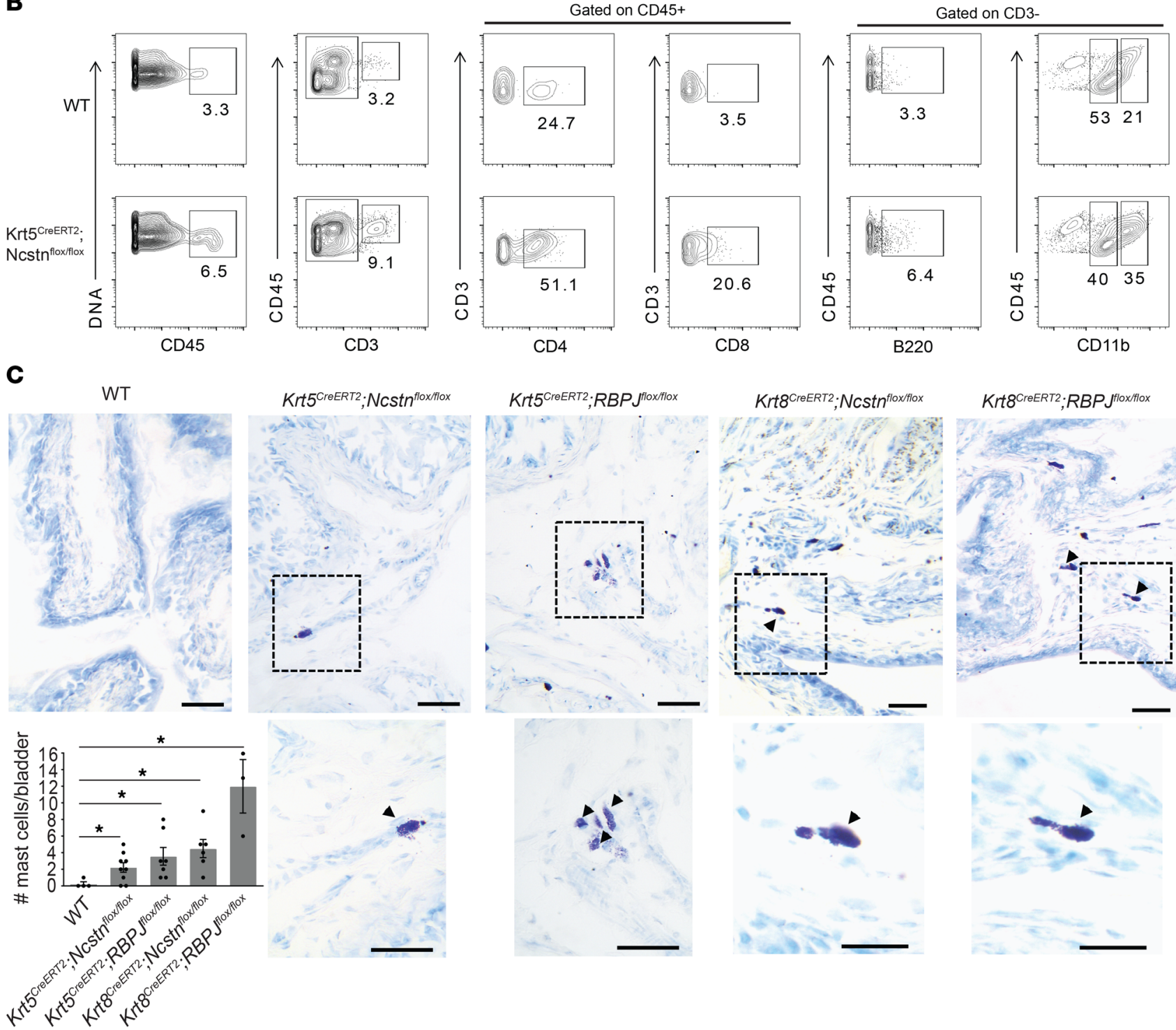

Figure 2. Notch inactivation leads to bladder inflammation. (A) Immunofluorescence on bladder sections from WT and Krt5 $5^{\mathrm{CreERT2}} \mathrm{Ncstn^{f/fl } \text { or }}$ Krt5 ${ }^{C r E R T 2} R B P f^{f / f l}$ mice with antibodies against KRT5 and the leukocyte marker CD45. Arrowheads in WT mice indicate positive stromal cells. Scale bars: $100 \mu \mathrm{m}$. DAPI was used as nuclear counterstain. (B) Analysis of mass cytometry data on leukocytic populations of bladder tissue from WT and Krt5 ${ }^{\text {CreeRT2 }}$ Ncst $n^{f l f f}$ mice ( $n=10$ in each group). Scatter dot plots from normalized data showing expression of CD45, CD3, CD4, CD8, B220, and CD11b. CD3 marks all T cells, CD4 detects helper T cells, CD8 is expressed in cytotoxic T cells, B220 is expressed in B cells, and CD11b is a common myeloid marker. Representative of 3 experiments. (C) Toluidine blue staining on WT and Notch-deficient mice of indicated genotypes marking mast cells. Arrowheads depict positive stromal mast cells that stain red/purple (metachromasia). Scale bars: $100 \mu \mathrm{m}, 25 \mu \mathrm{m}$ (insets). Scatter dot plot indicates 
toluidine blue-stained cells per 10 high-power fields (HPFs, original magnification, $\times 400$ ) ( $y$ axis) per bladder analyzed from each group. Data presented are mean \pm SEM. Number of mice analyzed: $4,9,7,6$, and 3 , respectively, for the 5 groups shown. ${ }^{*} P<0.05$. Student's $t$ test was used and $P$ values were corrected for multiple testing using the Benjamini-Hochberg method.

Whether increased permeability precedes the observed inflammation and possibly is causally involved in the inflammatory response is an interesting question. Among the integrin family members found downregulated, $\alpha_{4}$ integrin (Itga4) and its paralog $\alpha_{9}$ (Itga9) were at the top of the list in Notch-deficient mice (Figure 3D). Interestingly, Itga9 is a known direct target of Notch (37).

The role of $\alpha_{4}$ integrin in urothelial cell proliferation. It has been previously reported that mouse umbrella cell shedding caused by the administration of cyclophosphamide (CPP) leads to acute proliferation of basal cells (38), while a subset of Krt14-expressing progenitors are the first to respond (20). Even though mucosal sloughing and tissue damage in Notch-deficient bladders was extensive, we failed to detect a statistically significant increase in cell proliferation within the urothelial compartment (Figure 4A), despite the invariably evident hyperplasia, which in humans does not present substantial proliferation (39). Interestingly, the lack of proliferation is a hallmark of IC in spite of extensive umbrella cell exfoliation and severe inflammation (40). Integrin signaling has been implicated in key cellular processes, including regulation of cell proliferation (41) and tissue homeostasis (42), while immunofluorescence against activated (phosphorylated) focal adhesion kinase 1 (FAK1) (Supplemental Figure 5A) indicated that in the normal bladder urothelium integrin signaling is indeed active. The striking observation that, following Notch inactivation, both ECM components, such as fibronectin (Supplemental Figure 5B), as well as integrin receptors are downregulated prompted us to further investigate the functional role of integrins in urothelial cell proliferation. We focused on $\alpha_{4}$ integrin, which was the most downregulated member within the ECM-cell interaction pathway. In vitro clonogenic assays indicated that upon Itga4 knockdown mouse primary urothelial cells showed significantly reduced $(P<0.05)$ clonogenic potential (Figure $4, \mathrm{~B}$ and $\mathrm{C}$ ). In agreement with this, inhibition of FAK1, the downstream effector of integrin proliferative signaling, reduced the clonogenic potential of WT primary urothelial cells $(P<0.0001$; Figure $4 \mathrm{D})$. To investigate whether integrin activity is required for proliferation in vivo, we treated mice with an antibody against ITGA4 that blocks its interaction with ECM proteins, and subsequently with CPP, which as mentioned above is followed by acute basal cell proliferation. As Figure 4E indicates, blocking ITGA4 interaction with cognate ligands, and thus downstream signaling, led to a significant $(P<0.05)$ reduction of cell proliferation. Moreover, in vitro clonogenic assays indicated that blocking ITGA4 interaction significantly reduced the clonogenic potential $(P<0.01)$ of primary WT mouse urothelial cells (Figure $4 \mathrm{~F})$. Finally, constitutive exogenous expression of an Itga 4 cDNA in bladder explants from Notch-deficient mice (Krt$\left.5^{\text {CreERT2 }} N c s t n^{f / f f}\right)$ led to faster ex vivo growth highlighted by the fact that explants covered the filter surface and were scored after 3 days instead of the normal 7 days (Figure 4G). These data indicate that ITGA4 is required for urothelial cell proliferation both in vitro and in vivo and explain the absence of proliferation despite extensive tissue damage and immune cell infiltration.

Exogenous Notch activation reconstitutes urothelial integrity. To investigate whether Notch reactivation can rescue the observed phenotypes, we expressed in a Cre-dependent manner the intracellular portion of the human NOTCH1 receptor $\left(\mathrm{N}^{\mathrm{ic}}\right)$ under the control of the elongation factor 1a promoter (Ef1) (43). Notch activation on the ground of Ncstn loss substantially ameliorated the skin phenotype observed in Krt5 $5^{\text {CreERT2 }}$ $N c s t n^{f l f l}$ mice ( $n=9$; Supplemental Figure 2C). As Figure 5A indicates, mucosal sloughing was observed only focally while KRT20 polarity was reinstated in mice lacking Ncstn in basal cells. As expected, in mice $(n=5)$ lacking $R B P J$ in which Notch pathway activity cannot be reconstituted by reexpression of the constitutively active $\mathrm{N}{ }^{1}$, , mucosal sloughing was not rescued. This indicates involvement of the canonical Notch signaling in the process. Quantitative PCR analysis indicated that the expression levels of cell-cell and cell-ECM interaction components were also reconstituted, explaining the lack of evident tissue damage (Figure 5B). The reconstitution of integrin and ECM component expression in $\mathrm{Krt5}^{\mathrm{CreERT2}} \mathrm{Ncstn}^{\text {fl/fl}} \mathrm{EfI}^{\text {NIic }}$ likely led to reactivation of FAK1, as indicated by immunofluorescence with antibodies against the phosphorylated protein, which was not the case in $K r t 5^{\text {CreERT2 }} R B P J^{f / f l} E f 1^{\text {Nlic }}$ mice (Figure 5C). Moreover, mast cell infiltration was suppressed, and the suppressed levels were comparable to or indistinguishable from the WT mouse bladder levels only in bladders from $K r t 5^{\text {CreERT2 }} \mathrm{Ncstn}^{f / f l} \mathrm{EfI}^{\text {Nlic }}$ mice (Figure 5D). Both tissue integrity and KRT20 polarity were also reconstituted in mice in which the endogenous Notch pathway was inactivated and exogenously reactivated in the umbrella cell layer (Figure 5E). This led to a functional 
A

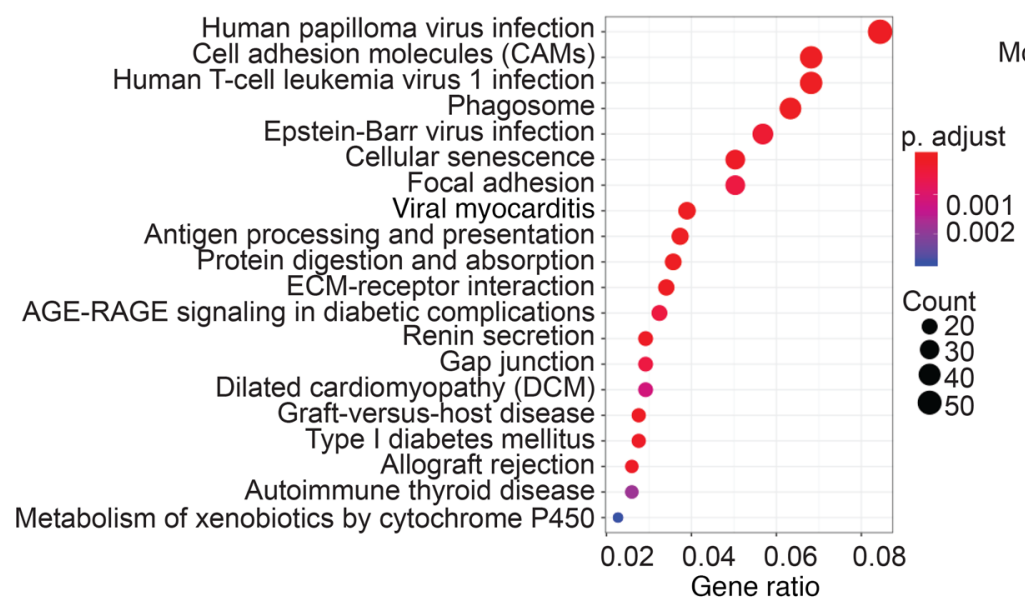

B

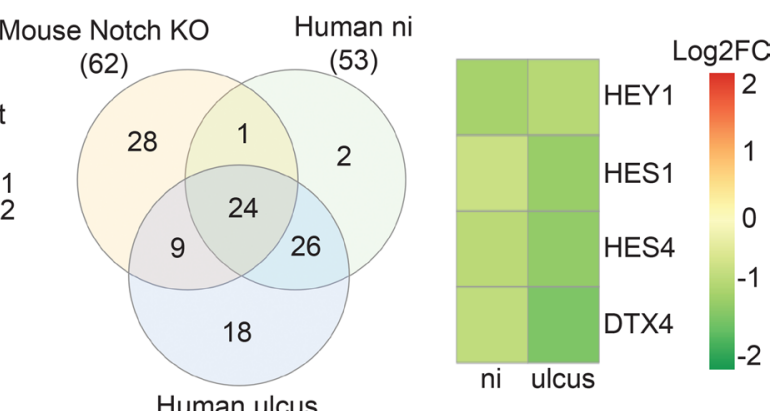

(77)
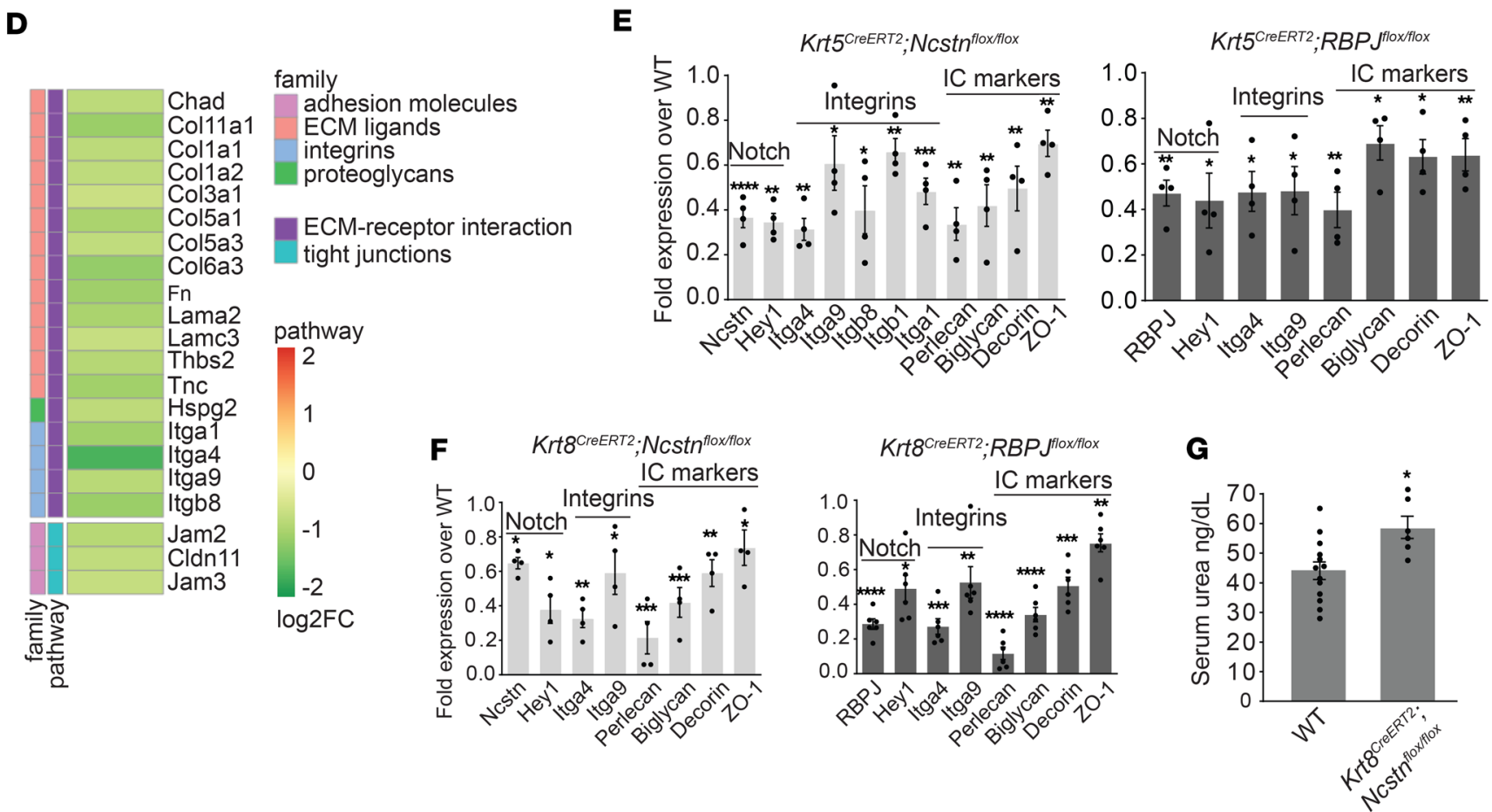

Figure 3. Molecular analysis of Notch-deficient mouse bladders. (A) GO network analysis of differentially expressed genes between WT ( $n=2$ ) and

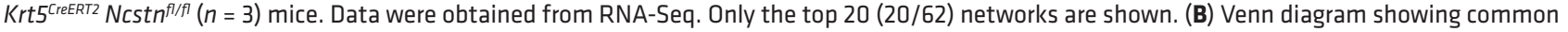
and independent networks affected in mouse bladders from Krt5 ${ }^{\text {CreERT2 }}$ Ncst ${ }^{f / f f}$ mice (RNA-Seq data) and human bladder biopsies from patients with IC corresponding to nonulcerative (ni) and ulcerative (ulcus) regions (Affymetrix expression data, GSE11783). A complete list of all processes as well as the common processes among mouse and human data can be found in Supplemental Table 1. (C) Heatmap depicting expression of canonical Notch pathway targets in the same data sets. (D) Heatmap indicating differential expression levels between WT and Krt5 $5^{\text {CreERT2 }}$ Ncstn ${ }^{\text {fl/fl }}$ mouse bladders of genes implicated in cell adhesion and cell-ECM interaction. Data were obtained from RNA-Seq. (E and F) Quantitative PCR analysis of indicated genes from urothelial cell RNA of Notch-deficient bladders. Expression is presented as ratio over WT mice. In all cases $n=4$ mice were analyzed. For $K r t 8^{\text {CreERT2 }} R B P f^{f / f l} n=6$ mice were analyzed. ${ }^{*} P<0.05$, ${ }^{* *} P<0.01,{ }^{* *} P<0.001$, and ${ }^{* * *} P<0.0001$. (G) Scatter dot plot indicating urea concentration (ng/dL) in serum from WT ( $n=$ 13) and $K r t 8^{\text {CreeRT2 }} \operatorname{Ncstn}^{f / f l}(n=6)$ mice. ${ }^{*} P<0.05$. For $\mathbf{E}, \mathbf{F}$, and $\mathbf{G}$, data presented are mean \pm SEM and Student's $t$ test was used.

urine-blood barrier as indicated by the normal urea levels measured in the serum of $\mathrm{Krtg}{ }^{\mathrm{CrERRT2}} \mathrm{Ncstn}^{\mathrm{Alf} f} \mathrm{EfI}^{\mathrm{Nii}}$ mice $(n=7$; Figure $5 \mathrm{~F})$. Collectively, these data indicate that the canonical Notch signaling through direct or indirect control regulates the expression of multiple components involved in cell-cell and cell-microenvironment crosstalk, while its loss leads to pleiotropic effects in urothelial integrity and cell proliferation. 
A

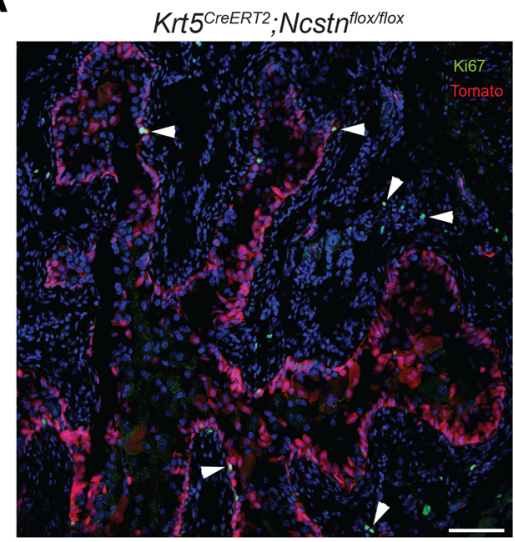

B

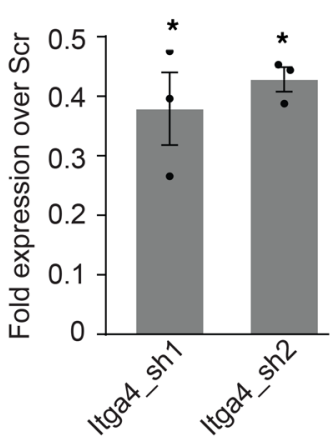

C

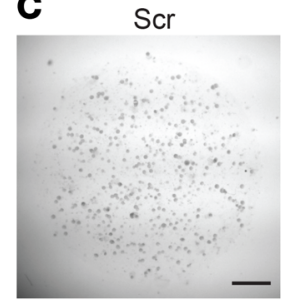

sh2
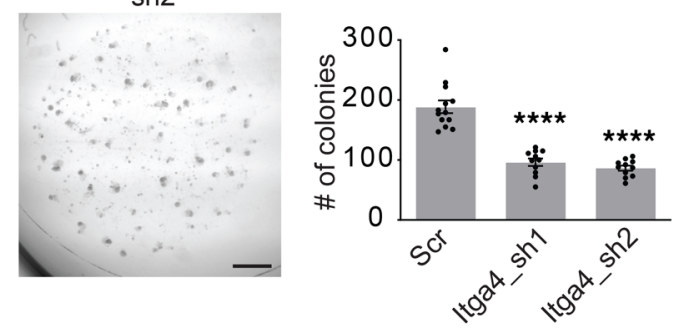

$\operatorname{sh} 1$

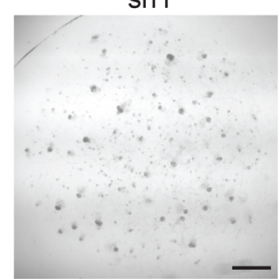

D

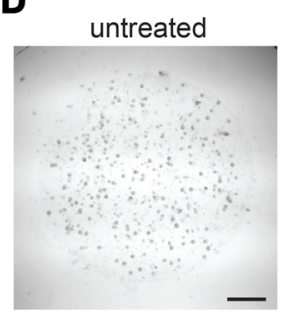

$1 \mathrm{uM}$
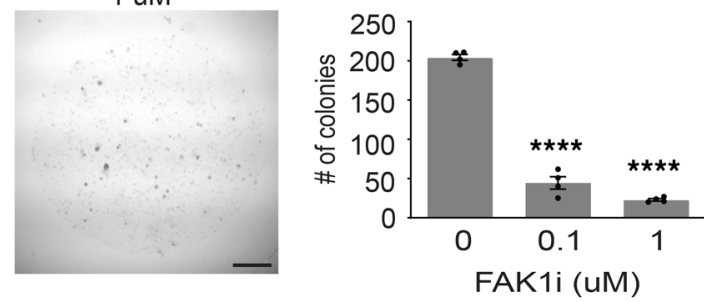

$\mathbf{F}$

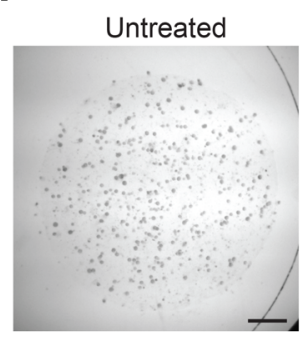

$0.1 \mathrm{uM}$

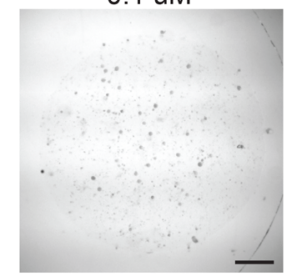

E

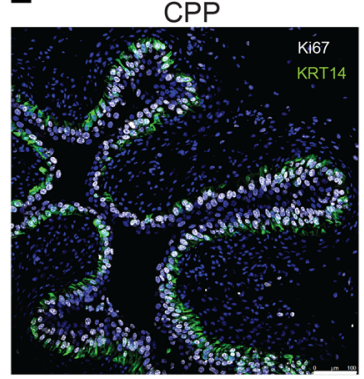

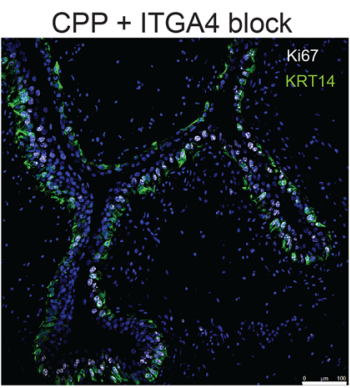

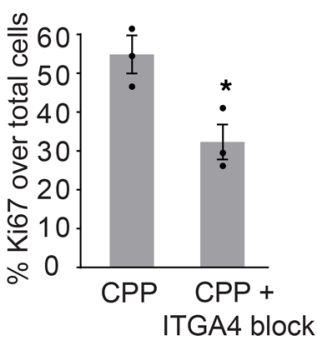

ITGA4 block
G
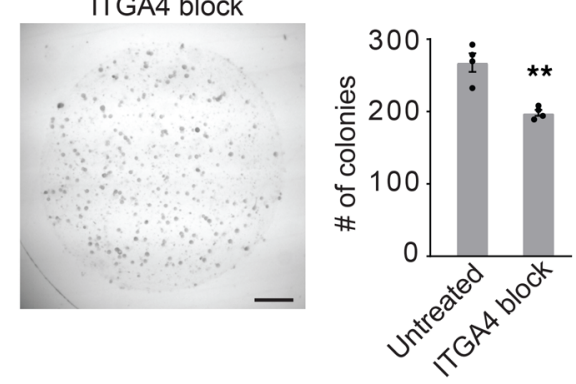

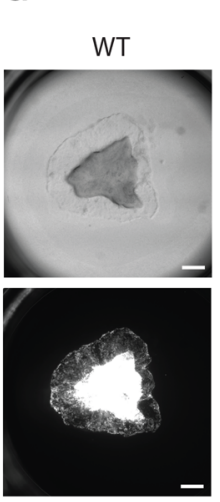

Krt5 CreERT2: Ncstn ${ }^{\text {floxfliox }}$
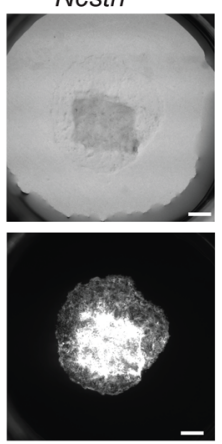

Krt5 ${ }^{\text {CreERT2 }}$;Ncstn floxflox + CMV/ltga4
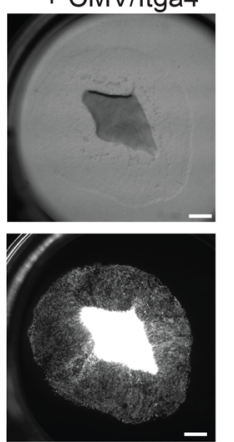

Figure 4. ITGA4 controls proliferation of bladder cells. (A) Immunofluorescence on Krt5 $5^{\text {creeRT2 }} \mathrm{Ncstn^{f/fl }}$ mouse bladders against the proliferation marker $\mathrm{Ki}-67$. Scale bar: $100 \mu \mathrm{m}$. Arrowheads indicate Ki-67+ cells. (B) Scatter dot plot indicating residual Itga4 expression levels in primary mouse bladder cells upon shRNA-mediated (sh1 and 2) knockdown of Itga4 expression. shRNAs were introduced through lentiviral infection. Expression is presented as ratio over control (cells infected with a scrambled shRNA sequence). In all cases $n=3$ experiments were analyzed. ${ }^{*} P<0.05$. (C) Representative images and scatter dot plot indicating clonogenic potential of primary bladder cell cultures on Matrigel isolated from C57BL/6 mice and infected with lentiviral vectors expressing control (Scr) or shRNAs against Itga4. On the $y$ axis is presented the number of colonies obtained from 10,000 cells. Results from 3 independent experiments are shown. ${ }^{* * *} P<0.0001$. Scale bar: $1 \mathrm{~mm}$. (D) Representative images and scatter dot plot indicating clonogenic potential of primary bladder cell cultures $(n=4)$ on Matrigel isolated from C57BL/6 mice treated with increasing concentration of the FAK1 inhibitor CSK2256098. On the $y$ 
axis is presented the number of colonies obtained from 10,000 cells. ${ }^{* * *} P<0.0001$. Scale bar: 1 mm. (E) Representative immunofluorescence images with antibodies against the proliferation marker Ki-67 and KRT14 in WT C57BL/6 mice challenged with CPP having received vehicle or an ITCA4 blocking antibody before the CPP administration. Scatter dot plot from $n=3$ mice for each group indicating proliferation index (percentage of Ki-67+ cells over total number of cells). ${ }^{*} P<0.05$. Scale bar: $100 \mu \mathrm{m}$. (F) Representative images and scatter dot plot indicating clonogenic potential of primary bladder cell cultures $(n=4)$ on Matrigel isolated from [57BL/6 mice and treated with either vehicle (untreated) or an ITCA4 blocking antibody. ${ }^{*} P<0.01$. Scale bar: 1 mm. (G) Representative whole-mount bright-field and fluorescence images (Tomato) of tissue explants from WT or Krt5 ${ }^{\text {CreERT2 }}$ Ncstn ${ }^{\text {fl/fl }}$ mice with or without constitutive exogenous expression of a mouse Itga 4 cDNA through lentiviral infection of the tissue explants. Scale bars: 1 mm. Explants were scored on day 3. For B-F, data presented are mean \pm SEM and Student's $t$ test was used. $P$ values were corrected for multiple testing using the Benjamini-Hochberg method (C and D). For C, D, and F, urospheres were photographed and scored on day 10. DAPI was used as nuclear counterstain in $\mathbf{A}$ and $\mathbf{E}$.

\section{Discussion}

The urinary bladder and particularly the umbrella cell layer are responsible for temporarily storing urine before excretion. Two functional properties characterize the urinary bladder: (a) the formation of an effective barrier that prevents highly concentrated ions and toxins from entering the bloodstream and (b) the ability to tremendously expand and shrink following alternating cycles of filling and voiding without epithelial tearing. These functional properties are served by a still understudied network of proteins participating in various cell-cell and cell-ECM structural complexes.

Although cancer is by far the most dangerous and life-threatening condition of the bladder, conditions associated with pelvic pain, incontinence, frequency of urination, and others also exist. IC/BPS alone is affecting as many as $2 \%$ of women (44), leading to substantially compromised quality of life. Neither the molecular basis nor effective treatments for these conditions are available. It is clear, however, that IC/BPS is the result of a leaky epithelium (45). We and others recently showed that mutations in Notch pathway components and NOTCH1 copy number loss are common events in human bladder cancer. Furthermore, genetic inactivation of the Notch signaling pathway in mice leads to urothelial tumorigenesis $(7,8)$. In this article we present compelling evidence for a broader role of Notch in controlling epithelial integrity and bladder function in mice. Our data implicate Notch for the first time to our knowledge in the transcriptional control of various components mediating cell-cell and cell-ECM interactions in the mouse bladder. Loss of active Notch signaling leads to downregulation of ECM components, integrins, and TJ proteins. Most likely, this concomitant reduction or loss of multiple structural proteins, which are important for urothelial integrity, compromised tissue integrity, leading to umbrella cell shedding into the lumen. Consequently, the barrier was weakened; urine penetrated the bladder wall, causing bladder inflammation, and possibly entered the bloodstream, leading to uremia. It is important to emphasize that this phenotype was observed following acute Notch inactivation based on tamoxifen-responsive Cre recombinase in adult mice. Maybe this could explain why we failed to observe this phenotype in our previous study (7), in which Notch inactivation was achieved through Cre recombinase expressed under the control of the Uroplakin 2 promoter from late embryogenesis onward.

Although Notch status in humans has not been implicated in conditions such as incontinence, frequency, urgency, or IC/BPS, mice lacking Notch activity in the urothelium present some similarities at both the histological and the molecular level with IC/BPS patients. This implies a possible role of Notch in bladder pathological conditions, beyond cancer, a hypothesis that could be further investigated in human patient samples. It is important to report that patients with IC are 3 times more likely to develop bladder cancer (46), which could of course relate to chronic inflammation.

In addition, our data uncover a potentially novel interplay between Notch and integrins, particularly $\alpha_{4}$ integrin, in cell proliferation. Our in vivo and in vitro data indicate that ITGA4 is important for urothelial cell proliferation even under acute damage conditions. Our previous experiments have shown that upon acute injury with CPP, which leads to umbrella cell exfoliation, basal Krt14-expressing cells respond by proliferating to regenerate the superficial layer and reconstitute the urine-blood barrier (20). Although the observed mucosal sloughing upon Notch genetic abrogation was not as extensive as that induced by CPP, nevertheless we expected that it would be sufficient to cause basal cell proliferation. This was not the case, however, which seems to contradict the tumorigenic effect of Notch loss. The pro-proliferative effect of Notch loss was more evident ex vivo, where Notch loss led to increased cell proliferation. Skin complications did not allow monitoring of $\mathrm{Krt}^{\text {CreERT2 }} \mathrm{Ncstn}^{\mathrm{fl} / \mathrm{fl}}$ and $K r t 5^{\text {CreERT2 }} R B P J^{f l f l}$ mice for periods beyond 1 month after tamoxifen injection, which would allow the development of dysplasia, carcinoma in situ, and invasive cancer, as we have previously reported (7). 
A
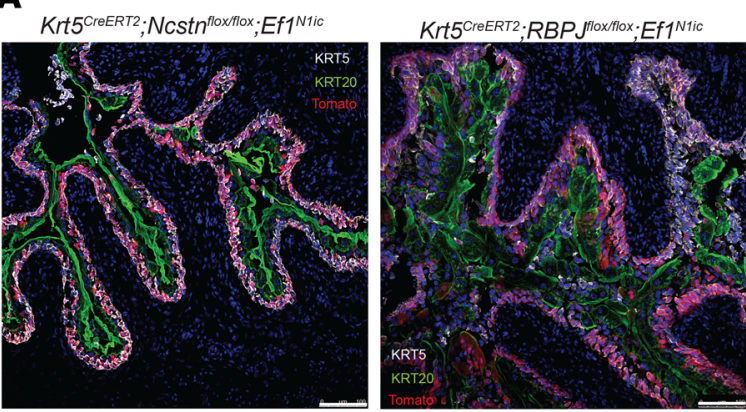

B

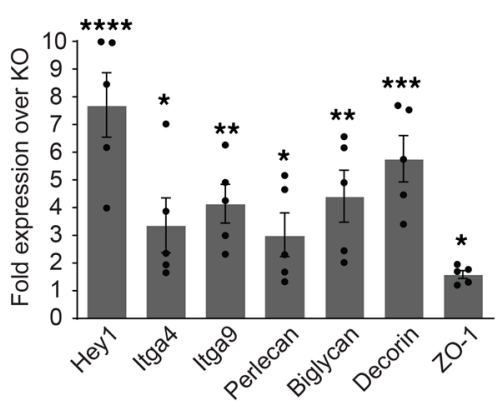

C

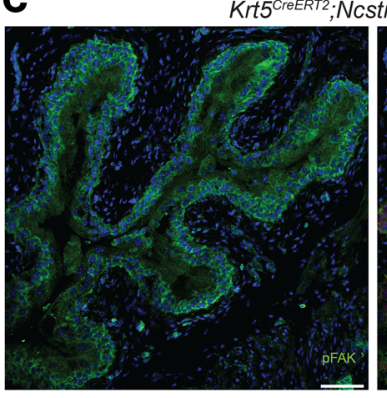

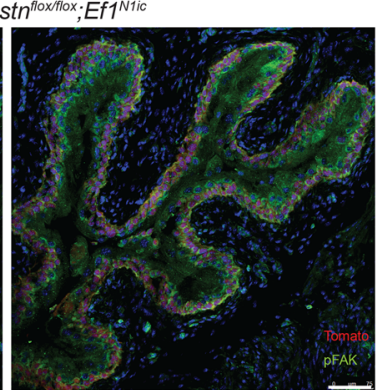

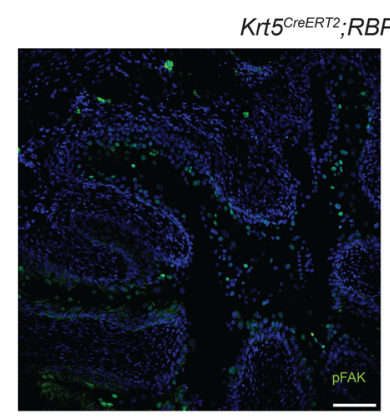

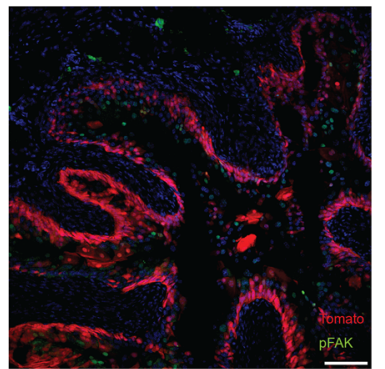

D

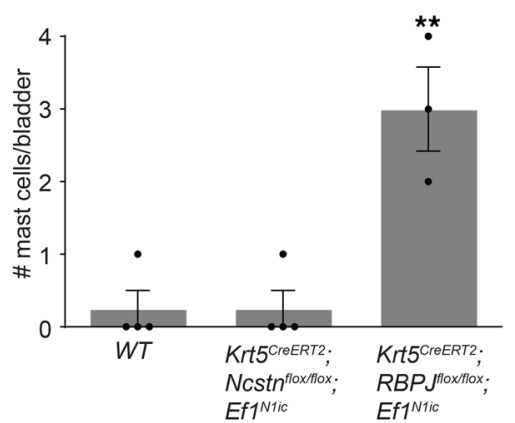

E

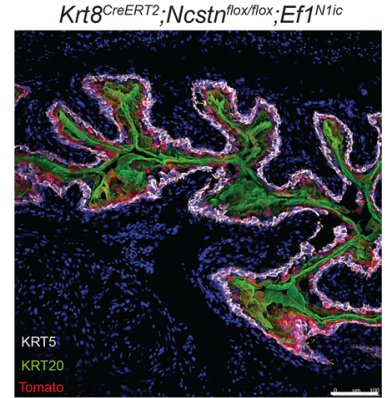

$\mathbf{F}$

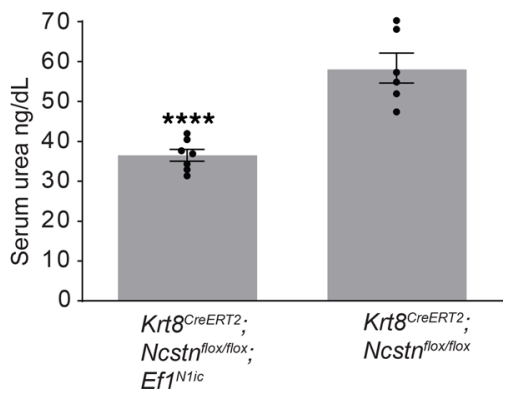

Figure 5. Reconstitution of the Notch pathway activity in mouse bladders rescues the epithelial integrity defects in vivo. (A)

Immunofluorescence with the indicated antibodies on bladder sections from mice lacking endogenous Notch activity, expressing a constitutively active form of the NOTCH1 receptor. Scale bar: $100 \mu \mathrm{m}$. (B) Quantitative PCR analysis of indicated genes from urothelial cell RNA of Krt5 $5^{\text {creeRT2 }}$ Ncstn ${ }^{f / f l}$ Ef1 ${ }^{\text {Nic }}$ mice $(n=5)$. Expression is presented as ratio over Krt5 ${ }^{\text {CreERT2 }}$ Ncstn ${ }^{f / f l}$ mice. ${ }^{*} P<0.05,{ }^{* *} P<0.01$, ${ }^{* * *} P<0.001$, and ${ }^{* * * *} P<0.0001$. (C)

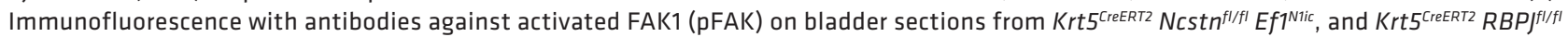
Ef1 ${ }^{\text {Niic }}$ mice. Scale bars: $75 \mu \mathrm{m}$ and $100 \mu \mathrm{m}$, respectively. (D) Scatter dot plot indicates the frequency of toluidine blue-stained cells per $10 \mathrm{HPFs}$ (original magnification, $\times 400$ ) ( $y$ axis) in bladders with reconstituted Notch signaling. Mice analyzed: 4 , 4, and 3 , respectively. The WT cohort is the same shown in Figure 2 C. ${ }^{* *} P<0.01$. (E) Immunofluorescence with the indicated antibodies on bladder sections from mice lacking endogenous Notch activity in the umbrella layer in which Notch activity has been reconstituted (Krt8 ${ }^{\text {creeRT2 }}$ Ncstn ${ }^{\text {fl/fl }}$ Ef ${ }^{\text {Nic }}$ ). Scale bar: $100 \mu \mathrm{m}$. (F) Scatter dot plot indicating urea concentration ( $\mathrm{ng} / \mathrm{dL}$ ) in serum from Notch-rescued and Notch-deficient $\left(n=7\right.$ and 6 , respectively). ${ }^{* * * *} P<0.0001$. For $\mathbf{B}, \mathbf{D}$, and F, data presented are mean value \pm SEM and Student's $t$ test was used. For D, $P$ values were corrected for multiple testing using the BenjaminiHochberg method. DAPI was used as nuclear counterstain in $\mathbf{A}, \mathbf{C}$, and $\mathbf{E}$.

These conditions are characterized by higher proliferation rates, which is not the case for simple hyperplasia, however (39). ITGA4, and possibly other ECM components, acting downstream of Notch seem to counteract the tumorigenic effects of its loss by restraining cell proliferation. We hypothesize that additional genetic or epigenetic events synergize with Notch loss and help overcome the antiproliferative effects of ITGA4 loss in vivo. Overall, this study uncovers a broad role of Notch in controlling multiple aspects of urothelial cell biology and points toward a general relation with human bladder pathophysiology. 


\section{Methods}

Mice. Previously described $K r t 5^{\text {CreERT2 }} R 26^{\text {tdTomato }}$ and $K r t 14^{C r e E R T 2} R 26^{\text {tdTomato }}$-bitransgenic mice (20) were crossed with $N c s t n^{f / f l}$ or $R B P J^{f l f l}$ homozygotes $(21,22,47)$ to produce $K r t 5^{\text {CreERT2 }} R 26^{\text {tdTomato }} N c s t n^{\text {flfl }}, K r t 14^{\text {CreERT2 }} R 26^{\text {tdTomato }}$ $N c s t n^{f / f l}, K r t 5^{C r e E R T 2} R 26^{\text {tdTomato }} R B P J^{l / f l}$, and $K r t 14^{\text {CreERT2 }} R 26^{\text {tdTomato }} R B P J^{f l / f l}$ offspring, respectively. Also, Krt8 CreERT2

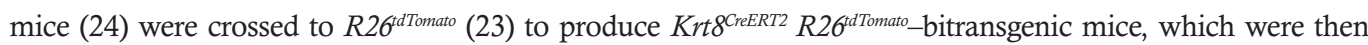
crossed to $N c s t n^{f l / f l}$ or $R B P J^{l / f l}$ to produce $K r t 8^{C r e E R T 2} R 26^{\text {tdTomato }} N c s t n^{f l f l}$ and $K r t 8^{C r e E R T 2} R 26^{\text {tdTomato }} R B P J^{l / f l}$ experimental mice as well. Upon crossing of $K r t 5^{\text {CreERT2 }} R 26^{t d T \text { mato }} N c s t n^{f l / f l}, K r t 5^{\text {CreERT2 }} R 26^{t d T \text { mato }} R B P J^{f / f l}$, and $K r t 8^{\text {CreERT2 }}$ $R 26^{\text {tdTomato }} N$ cst $^{\text {fl/fl}}$ offspring with Efla1 ${ }^{\text {Nlic }}$ mice $(43,48)$, we produced experimental offspring overexpressing the intracellular domain of NOTCH1 receptor under the control of the endogenous Ef1 locus. Male and female mice between 6 and 8 weeks of age were used for all experiments. For lineage-tracing studies all mice were injected with tamoxifen at 6 weeks of age, and $K r t 5^{\text {CreERT2 }} R 26^{\text {tdTomato }}, K r t 14^{\text {CreERT2 }} R 26^{\text {tdTomato }}$, and $K r t 8^{\text {CreERT2 }} R 26^{\text {tdTomato }}$ WT littermates were used as controls. For urothelial injury and in vivo blockade of ITGA4, as well as in vitro clonogenic assays, 8-week-old C57BL/6 male and female mice were used. In general, genetically modified mice were extensively backcrossed to a C57BL/ 6 background. Animals were housed in individually ventilated cages under specific pathogen-free conditions in full compliance with Federation of Laboratory Animal Science Associations recommendations in the Animal House Facility of the Biomedical Research Foundation of the Academy of Athens (BRFAA, Greece). All mice that were not generated by the authors, including WT C57BL/6 mice, were obtained from The Jackson Laboratory repository.

Lineage-tracing studies. Six-week-old experimental and WT mice of the genotypes described above were injected intraperitoneally with $3 \mathrm{mg}$ tamoxifen (MilliporeSigma) daily, for 5 consecutive days. Labeling of basal $\left(\mathrm{KRT}^{+}\right.$or $\left.\mathrm{KRT} 14^{+}\right)$and superficial $\mathrm{KRT}^{+}$cells was assessed within 1 month after the last tamoxifen injection.

Histology and antibodies. For immunofluorescence experiments, bladders were fixed in 4\% paraformaldehyde at $4^{\circ} \mathrm{C}$ for 2 hours, washed in PBS, placed in $30 \%$ sucrose overnight, and frozen in optimal cutting temperature compound (Tissue-Tek, Sakura Finetek). Frozen 12- $\mu$ m sections were obtained using a Leica (CM1950) cryostat. Sectioned tissues were fixed in 4\% paraformaldehyde for 5 minutes, washed 3 times in PBS, and blocked in 1\% bovine serum albumin with $0.1 \%$ Triton X-100 in PBS (PT) for 1 hour. Primary antibodies were added at the appropriate dilutions in PT, and tissue sections were incubated overnight at $4^{\circ} \mathrm{C}$ in humidified chambers. Tissues were incubated with secondary antibodies in PT for 2 hours at room temperature, after 3 washes with PT. Sections were counterstained with DAPI (MilliporeSigma) for 3 minutes, after 3 washes in PT, and finally mounted with Vectashield (Vector Laboratories). Catalog numbers and dilutions of antibodies used for immunofluorescence in this study were rabbit anti-Krt5 (BioLegend 905501, 1:1000), guinea pig anti-Krt20 (Progen Biotechnik GP-K20, 1:500), chicken anti-Krt14 (BioLegend 906001, 1:250), rabbit anti-Ki67 (Abcam ab15580, 1:150), rat anti-CD45 (BD Biosciences 550539, 1:500), rabbit anti-FAK1 phospho Y397 (Abcam ab81298, 1:100), mouse anti-fibronectin (BD 610077, 1:100), and rabbit anti-CD31 (Abcam ab28364, 1:200). For microscopic analysis of bladder vessels, tissues were fixed in formalin solution overnight, transferred to $70 \%$ ethanol, and embedded in paraffin. Five-micrometer sections were obtained, processed following standard protocol, and then used for H\&E stainings and immunofluorescence with anti-CD31 antibodies. All secondary antibodies (Alexa Fluor 488 AffiniPure donkey anti-rabbit IgG [H+L, 711-545-152], Cy5 AffiniPure donkey anti-rabbit IgG [H+L, 711-175-152], Alexa Fluor 488 AffiniPure donkey anti-guinea pig IgG [H+L, 706-545-148], Alexa Fluor 488 AffiniPure donkey anti-chicken IgY [IgG; H+L; 703-545155], Cy5 AffiniPure donkey anti-rat IgG [H+L, 712-175-153], and Alexa Fluor 488 AffiniPure donkey anti-mouse IgG [H+L, 715-545-150], all from Jackson ImmunoResearch) were used in a 1:500 dilution. $\mathrm{H} \& \mathrm{E}$ and toluidine blue stains were performed using standard histology procedures.

For IHC experiments, paraffinized sections were deparaffinized and processed according to standard procedures. Catalog numbers and dilutions of antibodies used for IHC were rabbit anti-cleaved Notch1 (Val1744) (Cell Signaling Technology 4147, 1:100), rabbit anti-phospho-p44/42 MAPK (ERK1/2) (Thr202/Tyr204) (Cell Signaling Technology 4370, 1:100), and anti-rabbit IgG, HRP-linked (Cell Signaling Technology 7074, 1:500).

CyTOF experiments. Single-cell suspensions were prepared from bladder tissues of $K r t 5^{\text {CreERT2 }} R 26^{\text {tdTomato }}$ $N c s t n^{f l f l}$ and $K r t 5^{C r e E R T 2} R 26^{t d T o m a t o}$ control mice (49). Briefly, tissues were collected, minced, and treated with dispase II and collagenase for 90 minutes at $37^{\circ} \mathrm{C}$. Then, cells were processed, stained, and fixed for CyTOF analysis using the Mouse Lymphocyte Basic Phenotyping Kit (Fluidigm) according to the manufacturer's guidelines. Cells were treated with cisplatin and Intercalator-Ir solution for live/dead and debris discrimination and stained with anti-CD45-147Sm (30-F11), anti-CD11b-148Nd (M1/70), 
anti-CD3e-152Sm (145-2C11), anti-CD8 $\alpha$-168Er (53-6.7), anti-CD4-172Yb (RM4-5), and anti-B220$176 \mathrm{Yb}$ (RA3-6B2) (included in Mouse Lymphocyte Basic Phenotyping Kit). Stained cells were then analyzed in a CyTOF Helios instrument (Fluidigm); data were normalized using CyTOF software v6.7 and analyzed with FlowJo software (Tree Star Inc.).

Urothelial tissue explant culture. The procedure has been previously described (20,50). After collection, bladders were rinsed in PBS and cut sagittally. The urothelium was separated from the muscle layer using forceps. Tissue fragments were spread onto $12-\mathrm{mm}$ diameter, $0.4-\mu \mathrm{m}$ pore size, polyethylene terephthalate Transwell filters (Corning) with the luminal side up and the lamina propria in contact with the filter. The culture medium used was a 1:1 mixture of MCDB153 and advanced Dulbecco's modified Eagle medium (MilliporeSigma) supplemented with $0.1 \mathrm{mM}$ ethanolamine (MilliporeSigma), $0.1 \mathrm{mM}$ phosphoethanolamine (MilliporeSigma), 0.5 $\mu \mathrm{g} / \mathrm{mL}$ hydrocortisone (MilliporeSigma), $5 \mu \mathrm{g} / \mathrm{mL}$ insulin (MilliporeSigma), $15 \mu \mathrm{g} / \mathrm{mL}$ adenine (MilliporeSig$\mathrm{ma}), 100 \mathrm{U} / \mathrm{mL}$ penicillin, and $100 \mu \mathrm{g} / \mathrm{mL}$ streptomycin. The explants were grown on the air-liquid interface. Media were changed every other day. For ex vivo CreERT2 activation, 4OHT (MilliporeSigma) was supplemented in the medium at $0.5 \mu \mathrm{M}$ for 12 hours. In the case of ex vivo overexpression of Itga 4 coding sequence, explants were transduced with lentivirus, and medium was also changed every other day.

$R N A$-Seq and analysis. Dissected urothelia separated from the muscularis propria from $K r t 5^{\text {CreERT2 }} R 26^{\text {tdTomato }}$ $N c s t n^{f / f l}$ and WT mice were frozen in liquid nitrogen and pulverized with a mortar and pestle, and total RNA was isolated using TRI Reagent (MilliporeSigma), according to the manufacturer's instructions. Library preparation for RNA-Seq was carried out in the Greek Genome Center (GGC) of the BRFAA. RNA-Seq libraries were prepared using the TruSeq RNA kit using $0.5 \mu \mathrm{g}$ of total RNA and were constructed according to Illumina's protocols. Equal amounts were mixed and run in the Illumina NextSeq500 in the GGC. Sequencing reads were aligned to the reference mouse genome GRCm38/mm10 using the algorithm bwa mem (v.0.7.17) (51). Alignment files were processed using the SAMtools tool kit (52). One WT sample was excluded from the analysis because of the low number of sequencing reads ( $<5$ million). The number of reads covering each transcript was calculated by the algorithm htseq-count (53) using the UCSC RefSeq (refGene) mm10 gene annotations for 43,252 genes. The estimation for dispersions and fold changes was conducted by the DESeq2 model (54) in R using the htseq-count data for transcripts covered by reads of minimum mapping quality 20. Differentially expressed genes were classified as those with a $\log _{2}$ fold change value less than -0.6 or greater than 0.6 and a Benjamini-Hochberg-corrected $P$ value of 0.05 .

Pathway enrichment analysis. In order to avoid any overestimation during pathway enrichment analysis, Kyoto Encyclopedia of Genes and Genomes (KEGG) pathway annotation was supervised by 2 R packages, gage (55) using the gene pathways set kegg.sets.mm and clusterProfiler (56) using the enrichKEGG function. $P$ values were corrected by Benjamini-Hochberg FDR method using the R function p.adjust. The GO network was designed using the function cnetplot of the R package clusterProfiler. Microarray analysis of the published Affymetrix data (GSE11783) was conducted as previously described (57).

Quantitative PCR with reverse transcription. Total urothelial RNA was isolated as described above. In the case of Matrigel-grown spheres, Matrigel was first digested using dispase II ( $5 \mathrm{mg} / \mathrm{mL})$ and spheres were collected by centrifugation. Complementary DNA samples were prepared using PrimeScript RT Reagent Kit (Takara), and quantitative PCR reactions were performed using KAPA SYBR FAST qPCR Master Mix (Kapa Biosystems), in a Roche LightCycler 96. Three independent biological samples were quantified in technical duplicates, and expression values were normalized to Gapdh. The quantitative PCR oligonucleotides used in this study are provided in Supplemental Table 2.

Urea assay kit. Isolated sera from experimental and WT mice were processed according to the manufacturer's instructions to measure urea levels using the QuantiChrom Urea Assay Kit (BioAssay Systems, DIUR-100).

Clonogenic assay. Bladders were dissected, everted using dissection forceps, and rinsed in PBS. Everted bladders were then placed in $5 \mathrm{mg} / \mathrm{mL}$ dispase II (MilliporeSigma) in growth medium and incubated at $37^{\circ} \mathrm{C}$ for 1 hour. Primary urothelial cells were collected, rinsed twice in PBS, and finally suspended in 1 mM EDTA in PBS at a density of 10,000 cells $/ \mu \mathrm{L}$, mixed with $40 \mu \mathrm{L}$ ice-cold Matrigel (Corning), and plated onto glass coverslips in 24-well tissue culture plates (20). Matrigel was allowed to solidify for 20 minutes at $37^{\circ} \mathrm{C}$, and a 1:1 mixture of MCDB153/advanced Dulbecco's modified Eagle medium (described above) and V79 lung fibroblast-conditioned medium (58) was added. Medium was changed every other day. For the effect of FAK1 inhibition in urothelial cultures, GSK2256098 (MedChemExpress, HY-100498) was added to the cultures at the indicated concentrations, and medium was changed every other day. 
shRNA knockdown and overexpression of Itga4. For the knockdown of Itga4, 2 shRNA sequences (GCAGACAACAATGGATATGTTccatggAACATATCCATTGTTGTCTGC and GCTCCAAATGTTAGTGTGAAAccatggTTTCACACTAACATTTGGAGC) were subcloned into pLKO.1/IRESegfp as previously described (59). Lentiviral supernatants were generated in HEK293T cells (ATCC) using standard procedures and concentrated using Amicon Ultra-15 centrifugal filters (20) (MilliporeSigma). Appropriate titers of shRNAs and scrambled sequence-expressing particles were used to transduce primary mouse bladder cells from C57BL/ 6 mice. Cells were incubated with the viral particles at $37^{\circ} \mathrm{C}$ for 1 hour, then mixed with $40 \mu \mathrm{L}$ Matrigel and plated. Lentiviral particles were maintained in the medium for 24 hours. Medium was changed every other day. In the case of Itga4 overexpression, the coding sequence was subcloned in the pLKO.1/IRESegfp backbone under the control of a CMV promoter. As described above, lentiviral supernatant was generated in HEK293T cells, concentrated, and used in appropriate titers for ex vivo transduction. Medium in explant cultures was also changed every other day.

Urothelial injury and in vivo ITGA4 blockade. Blocking anti-ITGA4 antibody (BioLegend, 103610) was administered by intraperitoneal injection to 8-week-old C57BL/6 mice at a single dosage of 5 $\mathrm{mg} / \mathrm{kg}$. Chemical injury of the urothelium was induced by intraperitoneal injection of a CPP (MilliporeSigma) solution in PBS (250 mg/kg) 48 hours after in vivo blockade. Bladders were collected 48 hours after CPP administration.

In vitro ITGA4 blockade. Total urothelial cells from 8-week-old C57BL/6 mice were isolated following the previously described protocol and plated on Matrigel in clonogenic densities (10,000 cells/well). The blocking anti-ITGA4 antibody was added to the cultures at a final concentration of $10 \mu \mathrm{g} / \mathrm{mL}$.

Microscopy and image processing. All images were captured on a Leica TCS SP5 inverted confocal, Leica HC, Leica DM IRE2, Leica DM LS2, or Nikon SM2800. Image processing and cell counts were performed using ImageJ.

Data availability. Data supporting the findings of this study are available within the article and its supplement. RNA-Seq data files can be found at Gene Expression Omnibus database (accession GSE140859).

Statistics. WT animals were randomly assigned into different groups. Group allocation and outcome assessment were not blinded. All quantitation was performed on at least 3 independent biological samples, using ImageJ software or the LAS AF 2.6.0. in the case of blood vessel surface quantitation. For quantitation of urospheres in Matrigel, a lower cutoff of $0.002 \mathrm{~mm}^{2}$ of sphere surface was used. Data presented are mean \pm SEM. Statistical analysis was performed using GraphPad Prism software v6. In 2-group comparisons, statistical significance was determined using a 2-tailed Student's $t$ test, considering a value of $P<0.05$ as significant. $P$ values were corrected for multiple testing using the Benjamini-Hochberg method. Statistical analysis of RNA-Seq and Affymetrix microarray data is described above.

Study approval. All procedures for the care and treatment of the animals were approved by the Institutional Committee on Ethics of Animal Experiments (BRFAA) and the Greek Ministry of Agriculture (Athens, Greece).

\section{Author contributions}

$\mathrm{AK}$ and VP conceived the study, designed all experiments, and analyzed data. VP and VB performed all experiments. VSD performed the computational analysis of RNA-Seq and Affymetrix microarray data. NP performed mass cytometry experiments. ISP performed histological evaluation. PM, EC, and EV helped with mouse breeding. AK wrote the manuscript.

\section{Acknowledgments}

We would like to thank V. Kostourou, P. Sideras, and A. Charonis for providing antibodies and reagents and thank G. Papafotiou and P. Karakaidos for critically reading the manuscript. Also, we would like to thank the GGC for next-generation sequencing; the BRFAA histology core facility for bladder tissue processing; and the BRFAA Imaging Unit for imaging and image processing. This work was supported by a Greek General Secretariat for Research and Technology "Excellence" grant (UTN_1799), a Fondation Santé Grant in Biomedical Sciences, a Worldwide Cancer Research grant (16/1217), and Horizon 2020 grants (732309 and 801347) to AK. This research was cofinanced by Greece and the European Union (European Social Fund) through the Operational Programme "Human Resources Development, Education and Lifelong Learning" in the context of the project "Strengthening Human Resources Research Potential via Doctorate Research" (MIS-5000432), implemented by the State Scholarships Foundation through a doctoral fellowship to VP. 
Address correspondence to: Apostolos Klinakis, Biomedical Research Foundation of the Academy of Athens, 4 Soranou Efesiou, Athens, Greece 11527. Phone: 30.210.6597069; Email: aklinakis@bioacademy.gr.

EC's present address is: Department of Cancer Biology and Genetics, College of Medicine, and Arthur G. James Comprehensive Cancer Center, The Ohio State University, Columbus, Ohio, USA.

1. Artavanis-Tsakonas S, Rand MD, Lake RJ. Notch signaling: cell fate control and signal integration in development. Science. 1999;284(5415):770-776.

2. Siebel C, Lendahl U. Notch signaling in development, tissue homeostasis, and disease. Physiol Rev. 2017;97(4):1235-1294.

3. Lobry C, Oh P, Aifantis I. Oncogenic and tumor suppressor functions of Notch in cancer: it's NOTCH what you think. J Exp Med. 2011;208(10):1931-1935.

4. Nowell CS, Radtke F. Notch as a tumour suppressor. Nat Rev Cancer. 2017;17(3):145-159.

5. Cancer Genome Atlas Network. Comprehensive genomic characterization of head and neck squamous cell carcinomas. Nature. 2015;517(7536):576-582.

6. Cancer Genome Atlas Research Network. Comprehensive molecular characterization of urothelial bladder carcinoma. Nature. 2014;507(7492):315-322.

7. Rampias T, et al. A new tumor suppressor role for the Notch pathway in bladder cancer. Nat Med. 2014;20(10):1199-1205

8. Maraver A, et al. NOTCH pathway inactivation promotes bladder cancer progression. J Clin Invest. 2015;125(2):824-830.

9. Khandelwal P, Abraham SN, Apodaca G. Cell biology and physiology of the uroepithelium. Am J Physiol Renal Physiol. 2009;297(6):F1477-F1501.

10. Acharya P, et al. Distribution of the tight junction proteins ZO-1, occludin, and claudin-4, -8 , and -12 in bladder epithelium. Am J Physiol Renal Physiol. 2004;287(2):F305-F318.

11. Varley CL, Garthwaite MA, Cross W, Hinley J, Trejdosiewicz LK, Southgate J. PPARgamma-regulated tight junction development during human urothelial cytodifferentiation. J Cell Physiol. 2006;208(2):407-417.

12. Slobodov G, Feloney M, Gran C, Kyker KD, Hurst RE, Culkin DJ. Abnormal expression of molecular markers for bladder impermeability and differentiation in the urothelium of patients with interstitial cystitis. J Urol. 2004;171(4):1554-1558.

13. Hauser PJ, Dozmorov MG, Bane BL, Slobodov G, Culkin DJ, Hurst RE. Abnormal expression of differentiation related proteins and proteoglycan core proteins in the urothelium of patients with interstitial cystitis. J Urol. 2008;179(2):764-769.

14. Hauser PJ, et al. Abnormalities in expression of structural, barrier and differentiation related proteins, and chondroitin sulfate in feline and human interstitial cystitis. J Urol. 2015;194(2):571-577.

15. Guan Y, et al. Redistribution of the tight junction protein ZO-1 during physiological shedding of mouse intestinal epithelial cells. Am J Physiol Cell Physiol. 2011;300(6):C1404-C1414.

16. Yu Y, et al. Alphavbeta6 is required in maintaining the intestinal epithelial barrier function. Cell Biol Int. 2014;38(6):777-781

17. Abrams $\mathrm{P}$, et al. The standardisation of terminology of lower urinary tract function: report from the Standardisation Sub-committee of the International Continence Society. Am J Obstet Gynecol. 2002;187(1):116-126.

18. Indra AK, et al. Temporally-controlled site-specific mutagenesis in the basal layer of the epidermis: comparison of the recombinase activity of the tamoxifen-inducible Cre-ER(T) and Cre-ER(T2) recombinases. Nucleic Acids Res. 1999;27(22):4324-4327.

19. Gandhi D, et al. Retinoid signaling in progenitors controls specification and regeneration of the urothelium. Dev Cell. 2013;26(5):469-482.

20. Papafotiou G, Paraskevopoulou V, Vasilaki E, Kanaki Z, Paschalidis N, Klinakis A. KRT14 marks a subpopulation of bladder basal cells with pivotal role in regeneration and tumorigenesis. Nat Commun. 2016;7:11914.

21. Klinakis A, et al. A novel tumour-suppressor function for the Notch pathway in myeloid leukaemia. Nature. 2011;473(7346):230-233.

22. Han H, et al. Inducible gene knockout of transcription factor recombination signal binding protein-J reveals its essential role in T versus B lineage decision. Int Immunol. 2002;14(6):637-645.

23. Madisen L, et al. A robust and high-throughput Cre reporting and characterization system for the whole mouse brain. Nat Neurosci. 2010;13(1):133-140.

24. Van Keymeulen A, et al. Distinct stem cells contribute to mammary gland development and maintenance. Nature. 2011;479(7372):189-193.

25. Sanfrancesco J, Jones JS, Hansel DE. Diagnostically challenging cases: what are atypia and dysplasia? Urol Clin North Am. 2013;40(2):281-293.

26. Duan M, et al. CD11b immunophenotyping identifies inflammatory profiles in the mouse and human lungs. Mucosal Immunol. 2016;9(2):550-563.

27. Larsen S, et al. Mast cells in interstitial cystitis. Br J Urol. 1982;54(3):283-286.

28. Theoharides TC, Kempuraj D, Sant GR. Mast cell involvement in interstitial cystitis: a review of human and experimental evidence. Urology. 2001;57(6 supp1 1):47-55.

29. Choi BH, et al. Differential perturbation of the interstitial cystitis-associated genes of bladder and urethra in rat model. Cell Cycle. 2017;16(8):749-758.

30. Gamper M, et al. Gene expression profile of bladder tissue of patients with ulcerative interstitial cystitis. BMC Genomics. 2009;10:199.

31. Lee SH. Intestinal permeability regulation by tight junction: implication on inflammatory bowel diseases. Intest Res. 2015;13(1):11-18.

32. Feng BS, et al. Expression of integrin alphavbeta6 in the intestinal epithelial cells of patients with inflammatory bowel disease. N Am J Med Sci. 2009;1(4):200-204.

33. Dowling J, Yu QC, Fuchs E. Beta4 integrin is required for hemidesmosome formation, cell adhesion and cell survival. J Cell Biol. 1996;134(2):559-572.

34. Georges-Labouesse E, Messaddeq N, Yehia G, Cadalbert L, Dierich A, Le Meur M. Absence of integrin alpha 6 leads to epi- 
dermolysis bullosa and neonatal death in mice. Nat Genet. 1996;13(3):370-373.

35. van der Neut R, Krimpenfort P, Calafat J, Niessen CM, Sonnenberg A. Epithelial detachment due to absence of hemidesmosomes in integrin beta 4 null mice. Nat Genet. 1996;13(3):366-369.

36. Raghavan S, Bauer C, Mundschau G, Li Q, Fuchs E. Conditional ablation of beta1 integrin in skin. Severe defects in epidermal proliferation, basement membrane formation, and hair follicle invagination. J Cell Biol. 2000;150(5):1149-1160.

37. Masià $\mathrm{A}$, et al. Notch-mediated induction of $\mathrm{N}$-cadherin and $\alpha 9$-integrin confers higher invasive phenotype on rhabdomyosarcoma cells. Br J Cancer. 2012;107(8):1374-1383

38. Farsund T, Dahl E. Cell kinetics of mouse urinary bladder epithelium. III. A histologic and ultrastructural study of bladder epithelium during regeneration after a single dose of cyclophosphamide, with special reference to the mechanism by which polyploid cells are formed. Virchows Arch B Cell Pathol. 1978;26(3):215-223.

39. Cina SJ, Lancaster-Weiss KJ, Lecksell K, Epstein JI. Correlation of Ki-67 and p53 with the new World Health Organization/International Society of Urological Pathology Classification System for Urothelial Neoplasia. Arch Pathol Lab Med. 2001;125(5):646-651.

40. Shie JH, Kuo HC. Higher levels of cell apoptosis and abnormal E-cadherin expression in the urothelium are associated with inflammation in patients with interstitial cystitis/painful bladder syndrome. BJU Int. 2011;108(2 pt 2):E136-E141.

41. Schwartz MA, Assoian RK. Integrins and cell proliferation: regulation of cyclin-dependent kinases via cytoplasmic signaling pathways. J Cell Sci. 2001;114(pt 14):2553-2560.

42. Livshits G, Kobielak A, Fuchs E. Governing epidermal homeostasis by coupling cell-cell adhesion to integrin and growth factor signaling, proliferation, and apoptosis. Proc Natl Acad Sci U S A. 2012;109(13):4886-4891.

43. Buonamici S, et al. CCR7 signalling as an essential regulator of CNS infiltration in T-cell leukaemia. Nature. 2009;459(7249):1000-1004.

44. Miller DC, Saigal CS, Litwin MS. The demographic burden of urologic diseases in America. Urol Clin North Am. 2009;36(1):11-27. 45. Parsons CL, Lilly JD, Stein P. Epithelial dysfunction in nonbacterial cystitis (interstitial cystitis). J Urol. 1991;145(4):732-735.

46. Keller J, Chiou HY, Lin HC. Increased risk of bladder cancer following diagnosis with bladder pain syndrome/interstitial cystitis. Neurourol Urodyn. 2013;32(1):58-62.

47. Sesele K, Thanopoulou K, Paouri E, Tsefou E, Klinakis A, Georgopoulos S. Conditional inactivation of nicastrin restricts amyloid deposition in an Alzheimer's disease mouse model. Aging Cell. 2013;12(6):1032-1040.

48. Weisberg SP, et al. ZFX controls propagation and prevents differentiation of acute T-lymphoblastic and myeloid leukemia. Cell Rep. 2014;6(3):528-540.

49. Lilly MA, et al. The murine bladder supports a population of stromal Sca-1+/CD34+/lin- mesenchymal stem cells. PLoS One. 2015;10(11):e0141437.

50. Kreft ME, Hudoklin S, Jezernik K, Romih R. Formation and maintenance of blood-urine barrier in urothelium. Protoplasma. 2010;246(1-4):3-14

51. Li H, Durbin R. Fast and accurate short read alignment with Burrows-Wheeler transform. Bioinformatics. 2009;25(14):1754-1760

52. Li H, et al. The Sequence Alignment/Map format and SAMtools. Bioinformatics. 2009;25(16):2078-2079.

53. Anders S, Pyl PT, Huber W. HTSeq--a Python framework to work with high-throughput sequencing data. Bioinformatics. 2015;31(2):166-169.

54. Love MI, Huber W, Anders S. Moderated estimation of fold change and dispersion for RNA-seq data with DESeq2. Genome Biol. 2014;15(12):550.

55. Luo W, Friedman MS, Shedden K, Hankenson KD, Woolf PJ. GAGE: generally applicable gene set enrichment for pathway analysis. BMC Bioinformatics. 2009;10:161.

56. Yu G, Wang LG, Han Y, He QY. clusterProfiler: an R package for comparing biological themes among gene clusters. OMICS. 2012;16(5):284-287.

57. Klaus B, Reisenauer S. An end to end workflow for differential gene expression using Affymetrix microarrays. F1000Res. 2016;5:1384.

58. Shin K, et al. Hedgehog/Wnt feedback supports regenerative proliferation of epithelial stem cells in bladder. Nature. 2011;472(7341):110-114.

59. Rampias T, et al. The lysine-specific methyltransferase KMT2C/MLL3 regulates DNA repair components in cancer. $E M B O$ Rep. 2019;20(3):e46821. 